\title{
Mesoscopic Coulomb Blockade in One-channel Quantum Dots
}

\author{
S. M. Cronenwett, S. M. Maurer, S. R. Patel, C. M. Marcus \\ Department of Physics, Stanford University, Stanford, California 94305 \\ C. I. Duruöz, J. S. Harris, Jr. \\ Department of Electrical Engineering, Stanford University, Stanford, California 94305
}

(submitted 17 July 1998)

PACS \#: 73.23.Hk, 72.23.-b, 73.20.Fz, 73.23.Ad

Signatures of "mesoscopic Coulomb blockade" are reported for quantum dots with one fully transmitting point-contact lead, $\mathcal{T}_{1}=1, \mathcal{T}_{2} \ll 1$. Unlike Coulomb blockade $(\mathrm{CB})$ in weaktunneling devices $\left(\mathcal{T}_{1}, \mathcal{T}_{2}<1\right)$, one-channel $\mathrm{CB}$ is a mesoscopic effect requiring quantum coherence. Several distinctive features of mesoscopic CB are observed, including a reduction in CB upon breaking time-reversal symmetry with a magnetic field, relatively large fluctuations of peak position as a function of magnetic field, and strong temperature dependence on the scale of the quantum level spacing.

Quantum dots provide a simple system to probe both quantum mechanical interference effects and the physics of electron-electron interactions. In dots weakly coupled to electronic reservoirs, i.e. when the two leads have transmissions $\mathcal{T}_{1}, \mathcal{T}_{2} \ll<1$, transport measurements show the influence of electron-electron interactions in the form of Coulomb blockade (CB), a classical effect which appears as strong peaks in dot conductance as an external gate voltage is swept, changing the dot potential [1]. Quantum interference in the weak-tunneling regime leads to random fluctuations of the CB peak height as a function of magnetic field and dot potential [2, 3]. In the opposite limit of several open channels per lead, $\mathcal{T}_{1}, \mathcal{T}_{2}>1$, quantum levels broadly 
overlap and a semiclassical picture of interfering trajectories of charged particles scattering through the dot can be used to describe transport. In the open regime, the physics of quantum interference is well understood, leading to coherent backscattering at zero magnetic field and universal conductance fluctuations [4]. Electron-electron interactions do not strongly affect transport in open dots, and no $\mathrm{CB}$ is observed. In the crossover regime, where the transmission of at least one point contact is unity, $\mathrm{CB}$ in dots disappears classically $[5,6]$ but can appear as a result of quantum interference, as recently discussed in Ref. [7]. Such "one-channel CB" will be the subject of this Letter.

In metallic dots, it is known both theoretically [8] and experimentally [9] that CB oscillations are washed out as the tunneling strength increases above $\mathcal{T}=1$ in either lead. In semiconductor quantum dots, experiments of $\mathrm{CB}$ in the strong tunneling regime have yielded varying results. Kouwenhoven, et al. [10], found that $\mathrm{CB}$ disappears when the transmission through one point contact reaches unity. However, Pasquier, et al. [11] report small CB oscillations up to $\mathcal{T}_{1}+\mathcal{T}_{2} \sim 3$, and Crouch, et al. [12] observed CB which decreases near $\mathcal{T}_{1}=1$, $\mathcal{T}_{2} \ll<$, but then increased again above $\mathcal{T}_{1}>1$. Flensberg [5] and Matveev [6] have shown theoretically that CB disappears at unity transmission when only inelastic processes are taken into account, appropriate in the limit $k T / \Delta \gg>1$, where $\Delta$ is the quantum level spacing. Recently, Aleiner and Glazman [7] extended the analysis to include elastic processes, finding for the particular case of "one-channel" transport $\left(\mathcal{T}_{1}=1, \mathcal{T}_{2} \ll 1\right)$ that although open-channel CB vanishes for purely inelastic transport, it persists due to coherent mechanisms for temperatures comparable to the quantum level spacing.

In this Letter, we investigate several novel features of one-channel CB which illustrate the interplay between quantum interference and electron-electron interactions. These are: (1) an enhancement of $\mathrm{CB}$ around zero magnetic field which, like coherent backscattering in open structures, can be understood in terms of the breaking of time-reversal symmetry; (2) a strong temperature dependence of $\mathrm{CB}$ on the scale of the quantum level spacing; (3) an enhanced 
correlation (though less than predicted theoretically) of conductance as a function of gate voltage, compared to the weak-tunneling regime, which results from cotunneling through many levels both on and off the CB peak; (4) large peak motion, predicted to be on the scale of the separation of CB peaks, as a function of magnetic field.

The existence of $\mathrm{CB}$ in the one-channel regime can be understood in terms of an effective scatterer at the location of the open lead that arises due to coherent trajectories reflected from the walls of the dot [7]. It is known that a real scatterer in a nearly-open lead ( $\mathcal{T}_{1}$ below unity) will cause classical CB $[5,6]$. Coherent processes give rise to a standing wave at the open lead which acts as such a scatterer, also giving rise to $\mathrm{CB}$ [7]. We emphasize that without coherence, $\mathrm{CB}$ should disappear in the fully transmitting one-channel case. At zero magnetic field, backscattering increases due to constructive interference of time-reversed paths, leading to stronger CB oscillations. As the magnetic field $B$ is increased beyond a characteristic field $B_{C}$ (where $B_{C}$ puts of order one flux quantum through the backscattered trajectories) the spectral power in the $\mathrm{CB}$ oscillations, $P_{C B}$, is predicted to decrease by a factor of 4 [7]. The requirement of coherence for $\mathrm{CB}$ in the one-channel dot implies a strong temperature dependence of $P_{C B}$ on the scale of the quantum level spacing $\Delta$ rather than the classical charging energy. Detailed calculations yield

$$
P_{C B}(B, T)=\frac{G_{R}{ }^{2}}{4} \Lambda(B) \alpha \frac{\Delta}{T}\left(\frac{\Delta}{E_{C}}\right)^{2} \ln ^{3}\left(\frac{E_{c}}{2 \pi k T}\right),
$$

where $\Lambda\left(B<<B_{C}\right)=4$ and $\Lambda\left(B>>B_{C}\right)=1, E_{C}$ is the classical charging energy, i.e. the energy to add a single electron to the dot, $\alpha \approx 0.207$ is a numerical factor, and $G_{R}$ is the conductance of the tunneling point contact [7]. Another consequence of the coherent nature of one-channel CB is that small changes in parameters such as device shape or magnetic field, which alter the interference pattern in the dot, can shift the position in gate voltage where the CB peak appears. 
Experimentally this shows up as a strongly $B$-dependent peak position, with excursions on the scale of the spacing between peaks.

We report measurements of two quantum dots [micrograph in Fig. 3(d), inset] fabricated using CrAu electrostatic gates $900 \AA$ above a two-dimensional electron gas (2DEG) on a GaAs/AlGaAs heterostructure. A multiple-gate design allows independent control of point contact conductances and dot shape via several shape-distorting gates. Both dots have an area of $0.5 \mu \mathrm{m}^{2}$ giving a level spacing $\Delta=2 \pi \hbar^{2} / \mathrm{m}^{*} A=14 \mu \mathrm{eV}$ ( $\mathrm{m}^{*}$ is the effective electron mass, $A$ is the dot area assuming a $100 \mathrm{~nm}$ depletion width). Measurements were made in a dilution refrigerator with an ac voltage bias of $\sim 5 \mu \mathrm{V}$ at $13.5 \mathrm{~Hz}$. The experimental temperature $T$ used throughout refers to the electron temperature, measured from the widths of CB peaks. At fridge base, $T=100 \mathrm{mK}$. Gate voltages can be related to dot energy through the ratio $\eta$, measured from the linear temperature dependence of the full-width-at-half-max of $\mathrm{CB}$ peaks in the weaktunneling regime at $T>\Delta$, where $e \eta(\mathrm{FWHM}) \approx 4.3 k_{B} T$. This ratio then converts peak spacing to charging energy, giving $e \eta($ peak spacing $)=E_{C}=260 \mu \mathrm{eV}$ for $\operatorname{dot} 1$ and $E_{C}=320 \mu \mathrm{eV}$ for dot 2 .

Figure 1 illustrates a number of novel features of one-channel transport. The rapid oscillations in conductance as a function of gate voltage are the $\mathrm{CB}$ oscillations. Comparing Figs. 1(a) and 1(b) shows that $\mathrm{CB}$ oscillations in the one-channel regime are considerably stronger at $B$ $=0 \mathrm{mT}\left(<<B_{C}\right)$ compared to $100 \mathrm{mT}\left(>>B_{C} \sim 20 \mathrm{mT}\right)$, unlike the weak-tunneling regime [Figs. 1(c, d)], where the strength of CB does not appear to depend on magnetic field. One-channel CB also shows large fluctuations of valley conductance due to large cotunneling contributions which are suppressed in the weak-tunneling regime.

The $B$ dependence of one-channel $\mathrm{CB}$ arising from the breaking of time-reversal symmetry can be studied quantitatively by evaluating the power spectral density $P_{g}(f)$ of the conductance, $g\left(V_{g}\right)$, at a number of different magnetic fields. The argument $f$ is the gate-voltage frequency in units of cycles/mV [13]. In both the one-channel and weak-tunneling regimes, $P_{g}(f)$ shows a clear peak around the $\mathrm{CB}$ frequency, $f_{C B}=\eta / E_{C}$, as seen in Figs. 2(a, b). In the one- 
channel regime, the $\mathrm{CB}$ peak in $P_{g}(f)$ has a clear maximum around $B=0$, whereas $\mathrm{CB}$ in the weak-tunneling regime is essentially independent of $B$. We define the $\mathrm{CB}$ power, $P_{C B}$, as the power in a window around the $\mathrm{CB}$ frequency in $P_{g}(f)$ (bracketed regions in Figs. 2(a, b)). The enhanced $\mathrm{CB}$ power around $B=0$ in the one-channel regime now appears as a peak at $B=0$ in the function $P_{C B}(B)$ [Fig. 2(c)], while $P_{C B}(B)$ is flat for weak tunneling [Fig. 2(d)]. We note that the width of the CB peak in $P_{g}(f)$ around $f_{C B}$ is broader and shows greater fluctuations in frequency in one-channel $\mathrm{CB}$ compared to the weak-tunneling regime. This implies a broader distribution of peak spacings in the one-channel regime, contrary to the predictions of Ref. [7], and remains an interesting open problem.

$\mathrm{CB}$ power normalized by its large- $B$ average, $p_{C B}(B)=P_{C B}(B) /\left\langle P_{C B}(B)\right\rangle_{B>B_{C}}$, and averaged over an ensemble of several dot shapes provides a useful quantity for comparing the $B$ dependence of $\mathrm{CB}$ in the one-channel and weak-tunneling regimes [Fig. 3(a)]. To compute these data, $P_{C B}(B)$ traces from seven (three) data sets in the one-channel (weak-tunneling) regime were normalized by the average value over $27 \mathrm{mT}<B<130 \mathrm{mT}$ [for two of the one-channel sets: 21 $\mathrm{mT}<B<60 \mathrm{mT}]$ and then averaged. The zero-field value, $\left\langle p_{C B}(0)\right\rangle \sim 5.3 \pm 0.5$, is somewhat larger than the predicted factor of 4 for reasons not yet understood. In the weak-tunneling regime $\left\langle p_{\mathrm{CB}}(0)\right\rangle \sim 0.7 \pm 0.2$, somewhat closer to unity than the zero-temperature theoretical value of $9 / 16$ [2], presumably due to decoherence. We note that a $B$-dependent real reflection in the point contact with maximum reflection at $B=0$ could lead to a spurious enhancement of $\mathrm{CB}$ power at $B=0$. To rule out this possibility, we have measured (in a separate device) the field dependence of the open point contact with the rest of the dot undepleted and find only very slight $B$ dependence with no distinct features on the 10-40 mT scale.

The temperature dependence of one-channel CB power is shown in Fig. 3(b) along with the no-free-parameters theory, Eq. (1), for both $B<<B_{C}$ and $B>>B_{C}$. Experiment and theory are roughly consistent up to $T \sim 300 \mathrm{mK}(k T \sim 2.5 \Delta)$, with good agreement in slope and the $T$ independence of the ratio $P_{C B}\left(B \ll B_{C}\right) / P_{C B}\left(B \gg B_{C}\right)$, and reasonable agreement in absolute 
magnitude given the lack of free parameters. Note the log scale on the vertical axes and that CB powers range over a factor of $\sim 100$. At temperatures above $\sim 400 \mathrm{mK}$, one-channel $\mathrm{CB}$ is strongly suppressed and the enhancement around $B=0$ disappears, as seen in both Figs. 2(b) and 2(c).

We have also investigated correlations in conductance as a function of $V_{g}$, $C\left(\delta V_{g}\right)=\left\langle g\left(V_{g}\right) g\left(V_{g}+\delta V_{g}\right)\right\rangle_{V_{g}}$ (average is over gate voltage) in the one-channel regime. Long correlations are expected in one-channel $\mathrm{CB}$ due to the significant contributions of cotunneling via many levels (of order $E_{C} / \Delta$ ) [14]. Figure 3(d) shows the discrete correlation function $C(\delta n)$, where $\delta n=0,1,2, \ldots$ acts as a peak index, defined for the one-channel CB regime as $C\left(\delta V_{g}\right)$ evaluated at the $\mathrm{CB}$ period, $\delta V_{g}=\delta n\left(E_{C} \eta \eta\right)$. In the weak-tunneling regime, $C(\delta n)$ is directly evaluated using sets of discrete peaks heights, $C(\delta n)=\left\langle g_{\max }(n) g_{\max }(n+\delta n)\right\rangle_{n}$, to avoid spurious correlations in $C\left(\delta V_{g}\right)$ caused by uniformly low valley conductance. The correlation length in the one-channel $\mathrm{CB}$ is considerably shorter than the theoretical value of $E_{C} / \Delta \sim 15$ peaks, and not significantly different than that of the weak-tunneling regime. This short correlation may be caused by changes in the energy spectrum of the dot as electrons are added with each successive CB peak. This suggests that the number of added electrons sufficient to scramble the dot spectrum is less than $E_{C} / \Delta$, consistent with similar conclusions based on the temperature dependence of peak correlations in the weak-tunneling regime [15].

Finally, we have investigated the predicted large-scale peak motion as a function of magnetic field in the one-channel $\mathrm{CB}$ regime. Whereas the weak-tunneling regime exhibits $\mathrm{CB}$ peak motion on the scale of the level spacing (once scaled to dot energy by $\eta$ ) [3], peak motion in the one-channel regime is expected to be of order $E_{C}$ [7]. An enhanced peak motion in $B$ for one-channel CB compared to weak-tunneling CB is seen in Fig. 4, although the effect is not as large as predicted theoretically. The standard deviation of peak motion about its average position is $0.09 E_{C}(\sim 2 \Delta)$ for one-channel CB [Fig. 4(a)], compared to $0.02 E_{C}(\sim 0.5 \Delta)$ in the weaktunneling regime [Fig. 4(b)], the latter consistent with previous measurements [3]. Large-scale 
motion of CB peaks in the strong-tunneling regime is the subject of ongoing investigations.

In summary, we have presented measurements of mesoscopic Coulomb blockade which arises due to quantum coherence in a quantum dot with one fully transmitting channel $\left(\mathcal{T}_{1}=1, \mathcal{T}_{2}\right.$ $<<1)$. One-channel $\mathrm{CB}$ is enhanced in the presence time-reversal symmetry and has a strong temperature dependence on the scale of the quantum level spacing, consistent with theory [7]. Correlation of conductance in gate voltage appears limited to $\sim 3$ peaks, smaller than expected, perhaps as a result of changes in the energy spectrum of the dot upon adding electrons. The motion of mesoscopic CB peaks with magnetic field is significantly greater than in the weaktunneling regime, but smaller than expected theoretically.

We thank I. Aleiner, L. Glazman, A. Johnson, L. Kouwenhoven, and K. Matveev for valuable discussions. We acknowledge support from the ARO under DAAH04-95-1-0331, the ORN-YIP under N00014-94-1-0622, the NSF NYI and PECASE programs under DMR 9629180-1, the A. P. Sloan Foundation (Marcus Group), and JSEP under DAAH04-94-G-0058 (Harris Group). SMC was supported by an NSF Graduate Fellowship and SMM was supported by a Hertz Foundation Fellowship. 
1. Single Charge Tunneling, Proceedings of a NATO Advanced Study Institute, edited by H. Grabert and M. H. Devoret (Plenum, New York, 1992); L. P. Kouwenhoven, C. M. Marcus, P. L. McEuen, S. Tarucha, R. M. Westervelt, and N. S. Wingreen, in Mesoscopic Electron Transport, edited by L. L. Sohn, L. P. Kouwenhoven, and G. Schön (Kluwer, Dordrecht, 1997).

2. R. A. Jalabert, A. D. Stone, and Y. Alhassid, Phys. Rev. Lett. 68, 3468 (1992).

3. A. M. Chang et al., Phys. Rev. Lett. 76, 1695 (1996); J. A. Folk et al., Phys. Rev. Lett. 76, 1699 (1996). Second paper includes peak motion information.

4. C. W. J. Beenakker, Rev. Mod. Phys. 69, 731 (1997); Mesoscopic Quantum Physics, edited by E. Akkermans, G. Montambaux, J.-L. Pichard, and J. Zinn-Justin (North Holland, Amsterdam, 1995).

5. K. Flensberg, Phys. Rev. B 48, 11156 (1993).

6. K. A. Matveev, Phys. Rev. B 51, 1743 (1995); A. Furusaki and K. A. Matveev, Phys. Rev. Lett. 75, 709 (1995).

7. I. L. Aleiner and L. I. Glazman, Phys. Rev. B 57, 9608 (1997).

8. D. S. Golubev et al., Phys. Rev. B 56, 15782 (1997); P. Joyez, D. Esteve, and M. H. Devoret, Phys. Rev. Lett. 80, 1956 (1998).

9. P. Joyez et al., Phys. Rev. Lett. 79, 1349 (1997).

10. L. P. Kouwenhoven et al., Z. Phys. B 85, 367 (1991).

11. C. Pasquier et al., Phys. Rev. Lett. 70, 69 (1993).

12. C. H. Crouch et al., Superlatt. and Microstr. 20, 377 (1996).

13.Power spectra are computed from half-overlapping segments of 256 points with Hanning windowing, normalized for each magnetic field so that the one-sided sum over frequencies equals $\operatorname{var}(g)$. See: W. H. Press, S. A. Teukolsky, W. T. Vetterling, and B. P. Flannery, Numberical Recipes in C, (Cambridge University Press, Cambridge, 1992).

14. I. L. Aleiner and L. I. Glazman, Phys. Rev. Lett. 77, 2057 (1996); S. M. Cronenwett et al., Phys. Rev. Lett. 79, 2312 (1997).

15. S. R. Patel et al., preprint (submitted), (1998). 

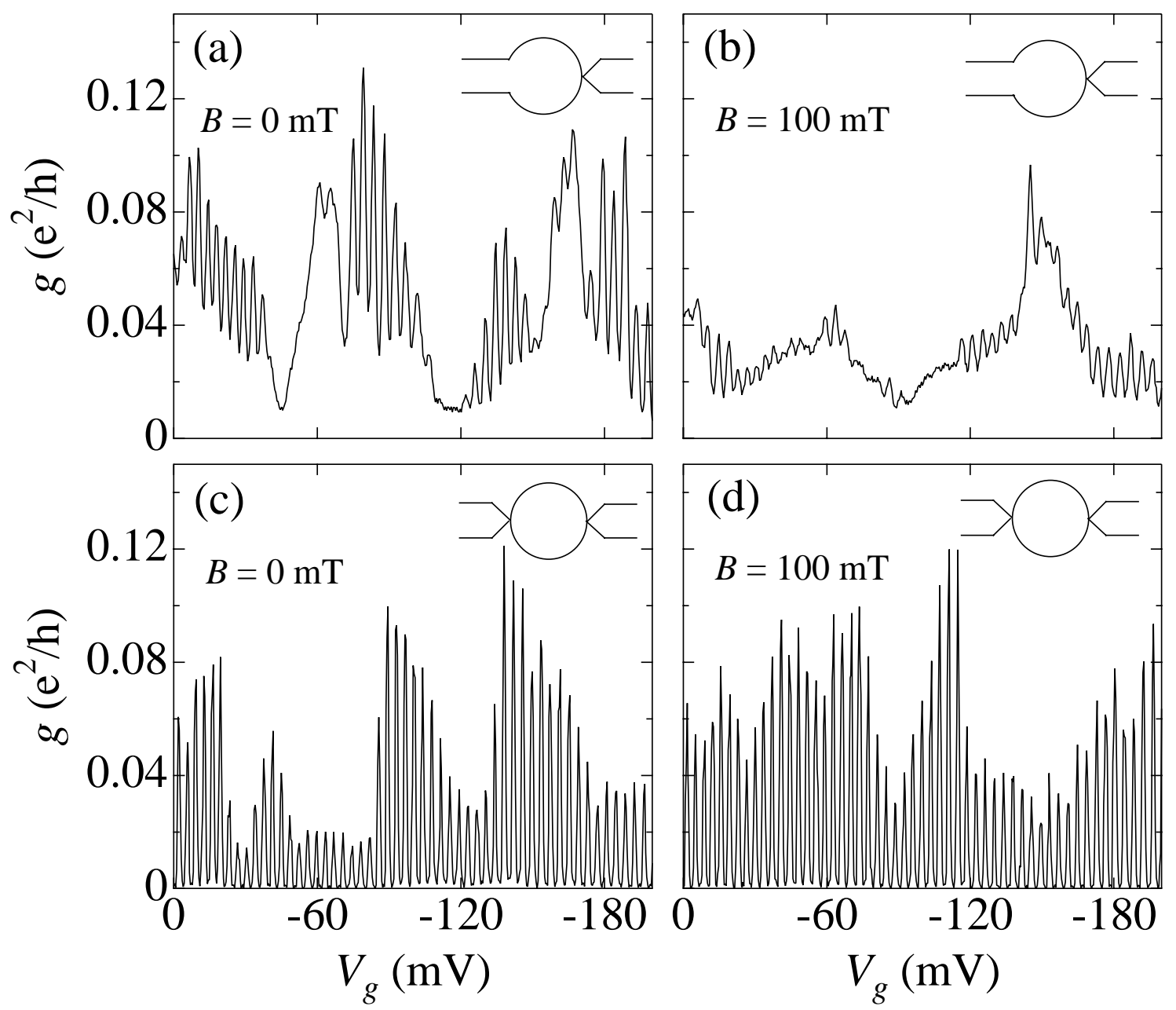

Fig. 1: Conductance showing Coulomb blockade (CB) oscillations as a function of gate voltage, $V_{g}$, in the one-channel regime $(\mathrm{a}, \mathrm{b})$ and weak-tunneling regime $(\mathrm{c}, \mathrm{d})$ at $B=0 \mathrm{mT}\left(<B_{C} \sim 20\right.$ $\mathrm{mT}$ ) and $B=100 \mathrm{mT}$ ( $>B_{C}$ ) (dot 1). One-channel $\mathrm{CB}$ is stronger at $B=0 \mathrm{mT}$ compared to $B=$ $100 \mathrm{mT}$, unlike weak-tunneling CB. 

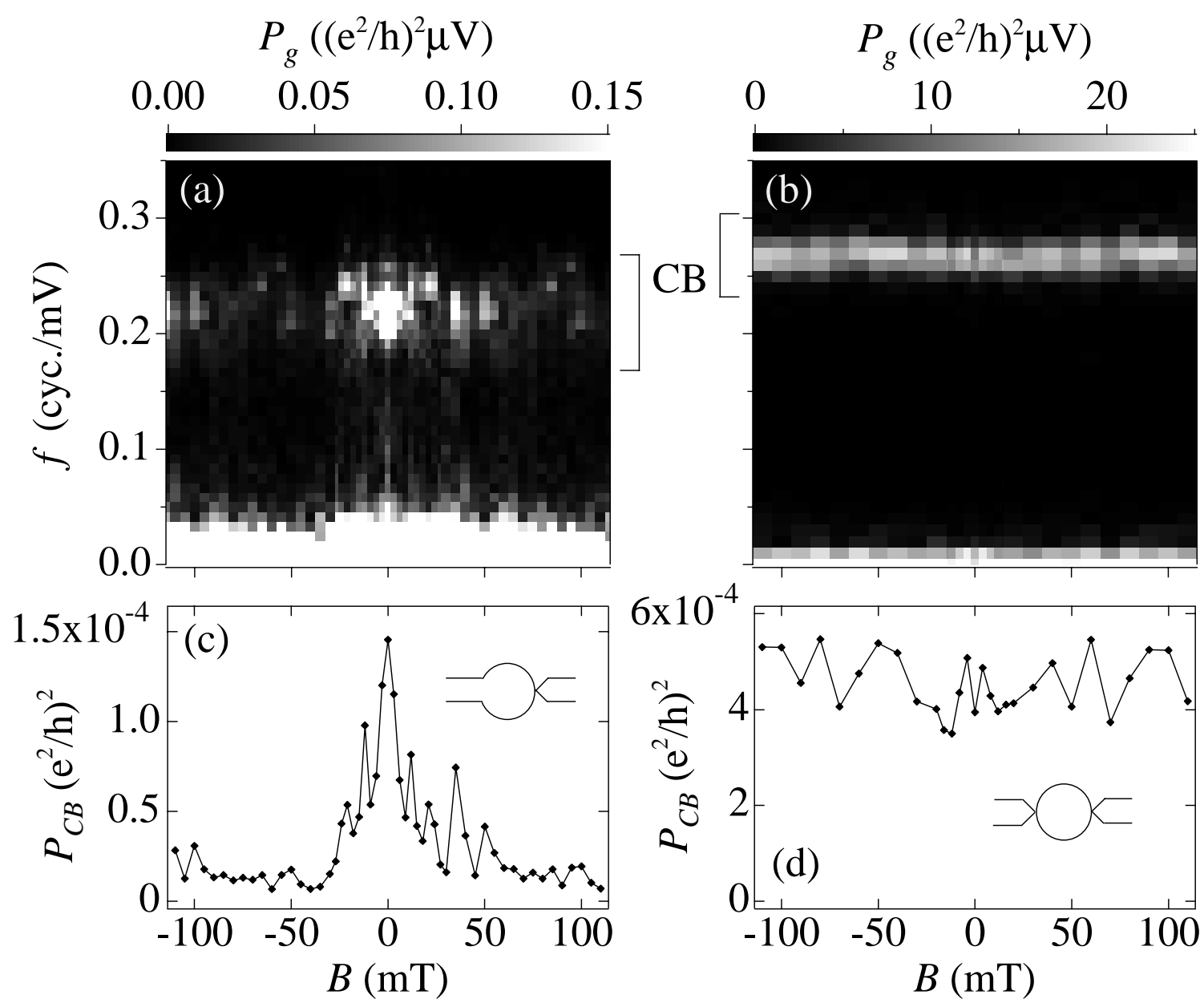

Fig. 2: $(\mathrm{a}, \mathrm{b})$ Grayscale plots of the power spectral density, $P_{g}$, as a function of gate-voltage frequency $f$ (cycles/mV) and magnetic field $B$ for (a) one-channel $\mathrm{CB}$ and (b) weak-tunneling $\mathrm{CB}$ (dot 1). The dominate frequency of $\mathrm{CB}$ oscillations is within the bracketed region marked " $\mathrm{CB}$ " in each plot. In the one-channel regime (a), the bright structure at $B \sim 0$ at the $\mathrm{CB}$ frequency indicates stronger $\mathrm{CB}$ at zero field. No corresponding field dependence of $\mathrm{CB}$ is seen for weak tunneling (b). At each magnetic field, the power within the bracketed region defines $P_{C B}$ $(B)$, the $\mathrm{CB}$ power. (c, d) $P_{C B}(B)$ for data in (a) and (b). Again, the zero-field enhancement of CB in the one-channel regime is seen as a peak in $P_{C B}(B)$ around $B=0$. 

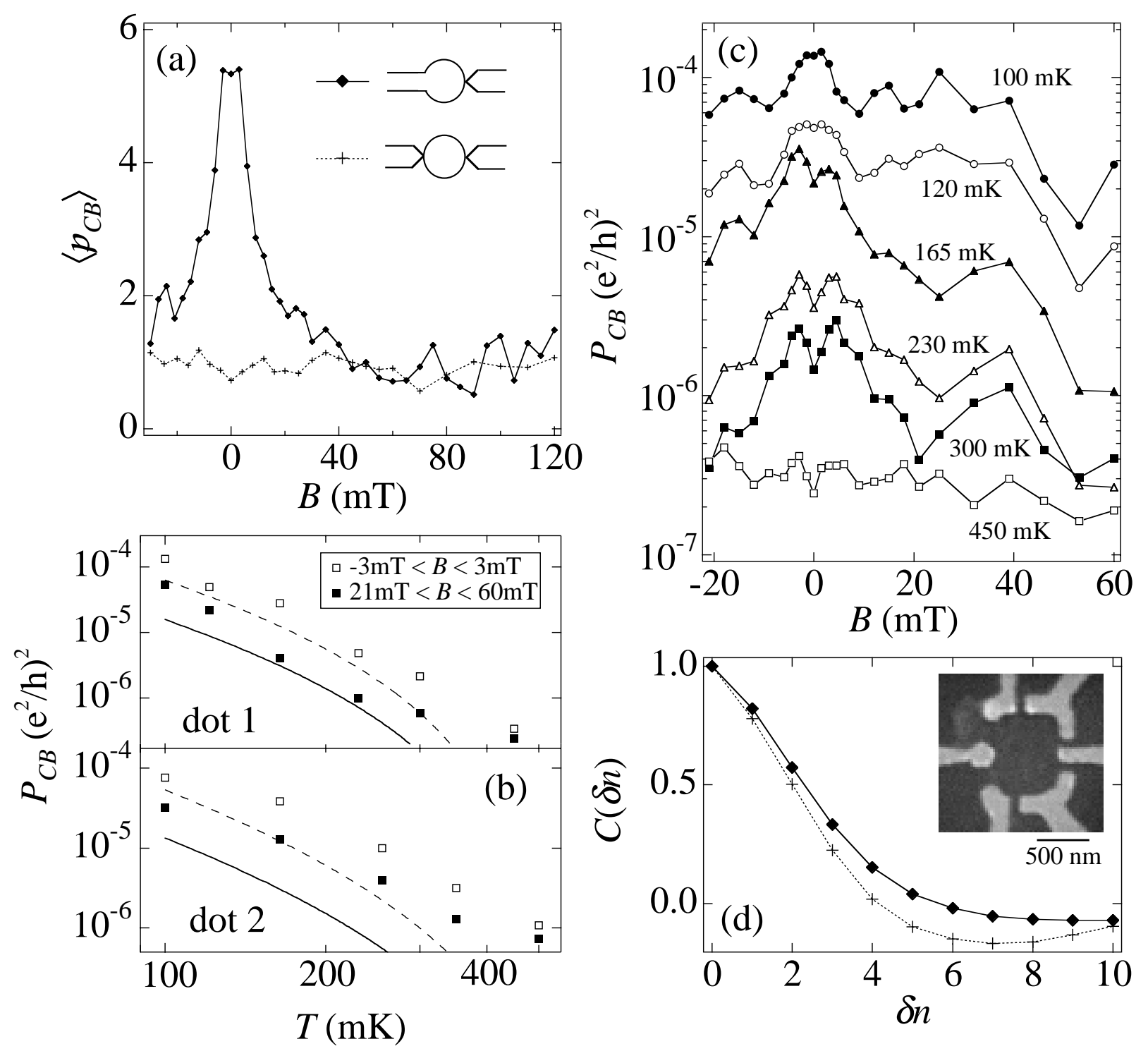

Fig. 3: (a) Magnetic field dependence of $\mathrm{CB}$ oscillations for the one-channel and weak-tunneling regimes given by the $\mathrm{CB}$ power normalized to its $B \gg B_{C}$ value and averaged over shape configurations, $\left\langle p_{C B}(B)\right\rangle$ (dot 1). Uncertainties are $\sim 10 \%$ for the one-channel regime and $\sim 30 \%$ for the weak-tunneling regime. (b) $\mathrm{CB}$ power, $P_{C B}(B)$, as a function of temperature, averaged over field ranges $-3 \mathrm{mT}<B<3 \mathrm{mT}$ (open squares) and $21 \mathrm{mT}<B<60 \mathrm{mT}$ (solid squares), along with theory (Eq. (1)) for $B<<B_{C}$ (dashed curve) and $B \gg B_{C}$ (solid curve). (c) $P_{C B}(B)$ decreases strongly with increasing temperature from $100 \mathrm{mK}$ (top curve) to $450 \mathrm{mK}$ (bottom curve) in dot 1 . The zero-field peak in $P_{C B}(B)$ persists up to $\sim 400 \mathrm{mK}$. (d) Magnetic-fieldaveraged autocorrelation function, $C(\delta n)$, of CB conductance oscillations in gate voltage $V_{g}$ in units of peak number $\delta n$, where $\delta V_{g}=\delta n\left(E_{C} \eta\right)$ for dot 1 . The correlation length for the onechannel $\mathrm{CB}$ regime (diamonds) is $\sim 3$ peaks, slightly larger, but comparable to the weaktunneling case (crosses). Inset: SEM micrograph of dot 1. 


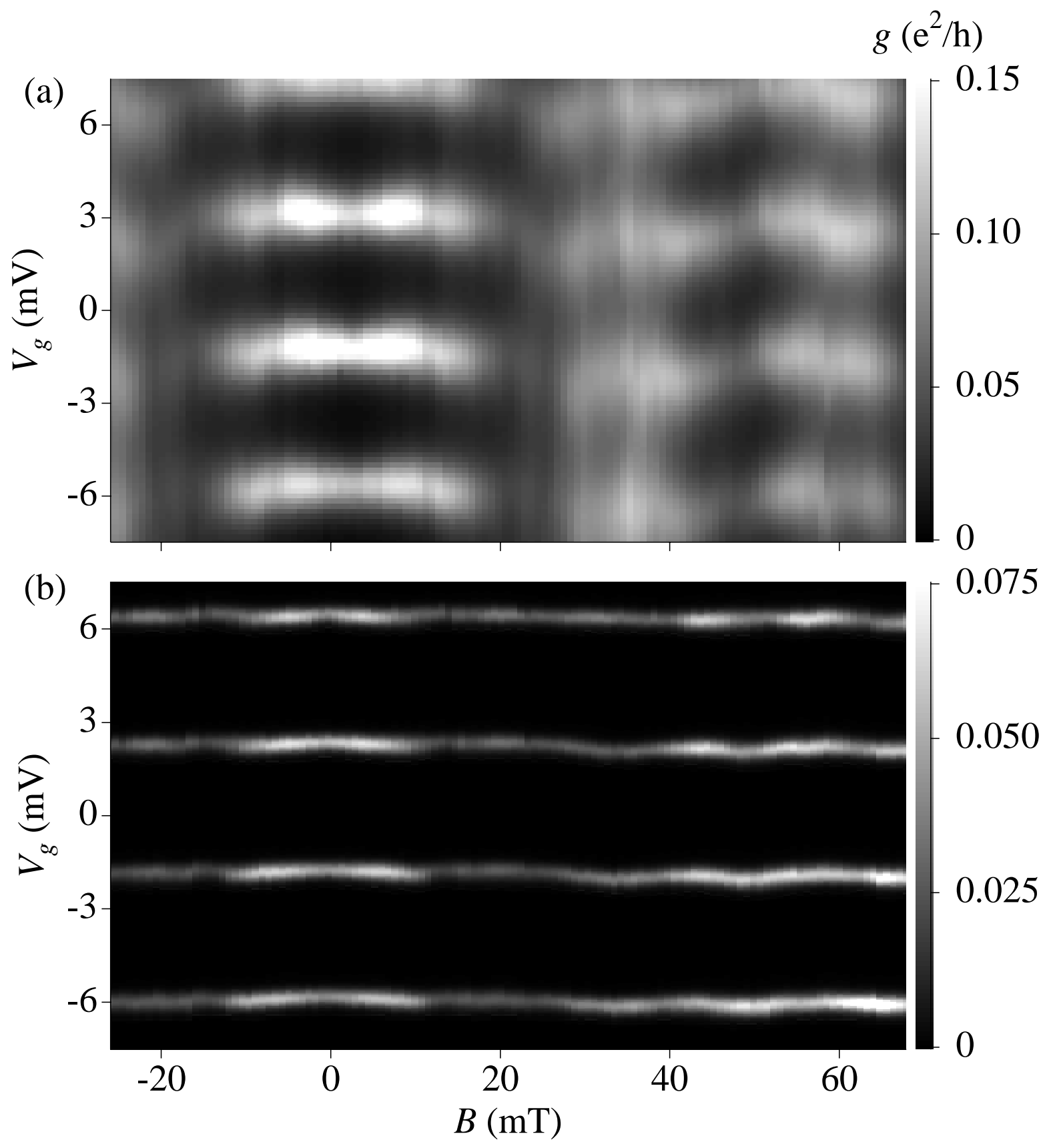

Fig. 4: Grayscale plots of conductance versus gate voltage $V_{g}$ and magnetic field $B$ shows larger fluctuations of $C B$ peak position as a function of $B$ in the one-channel regime (a) than in the weak-tunneling regime (b). Note symmetry in $B$ of $\mathrm{CB}$ peak height and position (dot 1 ). 